\title{
Spontaneous subdural hematoma according to sudden unexpected death
}

\begin{abstract}
Sudden deaths are defined as, "Deaths occurring within 24 hours of the onset of the symptoms". The subdural hematoma, which is among the causes of central nervous system-related death that has a large prevalence among sudden unexpected causes of death, is often due to the injury of the cerebral bridge veins to the head trauma. About two percent to 6.7 percent of acute subdural hematomas constitute spontaneous acute subdural hematomas. A 53-year-old man was admitted to the hospital because of severe headache, was referred to an upper center on the development of unconsciousness while continuing treatment in the observation room, and died in spite of all the interventions performed at the center. In the constructed autopsy; a subdural hematoma with a thickness of 2-3 $\mathrm{mm}$ in the entire posterior fossa on the brain-cerebellum surface where the skull bones and both temporal muscle groups are intact. A spontaneous subdural hematoma was diagnosed as a subdural hematoma because of the presence of a severe headache in the patient, a cardiac prosthesis in his autopsy, an anticoagulant medication, and no traumatic lesion under the scalp and bone. It should be considered that subdural hematomas may develop spontaneously, with aspirin, heparin, warfarin use, even in simple movements that are not related to head trauma.
\end{abstract}

Volume 6 Issue 2 - 2018

\author{
Eren Akgündüz,' Kenan Kaya,' Sila Aslan, ${ }^{2}$ \\ Ebubekir Burak Çelik,' Necmi Çekin ${ }^{1,2}$ \\ 'Ministry of Justice, Council of Forensic Medicine, Turkey \\ ${ }^{2}$ Department of Forensic Medicine, Cukurova University School \\ of Medicine, Turkey
}

Correspondence: Kenan Kaya, Ministry of Justice, Council of
Forensic Medicine,Adana, Turkey, Tel +905066472403, Email k_kaya_7l@hotmail.com

Received: March 29, 2018 | Published: April II, 2018

Keywords: death, spontaneous, subdural hematoma, autopsy

\section{Introduction}

Sudden deaths are defined as: "Deaths occurring within 24 hours of the onset of the symptoms". If a person without a known disease is found dead or a person dies without a reason within a short period of time, the death of a person who has a known illness but who does not have a clinic to cause the illness is generally considered unexpected death by his or her relatives. According to the order of frequency, cardiovascular system, respiratory system, central nervous system and digestive system origin. Since death occurs in such a short period of time, the cause and mechanism of death is not explained. For this reason, an autopsy is necessary to explain the cause of death. Despite all studies in some deaths, the cause of death can not be determined and the rate of these cases is reported as five to 10 percent of the time. ${ }^{1,2}$

The subdural hematoma, which is among the causes of central nervous system-related death that has a large prevalence among sudden unexpected causes of death, is often due to the injury of the cerebral bridge veins to the head trauma. Arterial bleeding can also occur as a result. About two percent to 6.7 percent of acute subdural hematomas constitute spontaneous acute subdural hematomas. Although spontaneous subdural hematomas are rare, the mortality rate is between 60 percent and 76.5 percent. $^{3-5}$ In addition, the majority of cases with acute spontaneous subdural hematomas are men in the middle age group, the majority being hypertension. In these cases, the hematoma is often venous rather than arterial.

\section{Case presentation}

A 53-year-old man was admitted to the hospital because of severe headache. In the observation room, while the treatment was continuing, loss of consciousness developed, forwarded to an advanced center, and died in spite of all the interventions performed at the center. In the constructed autopsy; a subdural hematoma with a thickness of two to three milimeters in the entire posterior fossa on the brain surface where the skull bones and both temporal muscle groups are intact (Figure 1), the brain and cerebellum sections have no pathology other than congestion and edema except on the head examination of ecchymoses and hematomas under the scalp that the base bones are firm, on the chest; the coronary vessel lumen was mild to moderate on the left and right, the aortic valve was replaced with a metal cap, the myocardial sections were not macroscopically scarred and the aortic mild atheromatous plaques were parallel to the macroscopic findings.

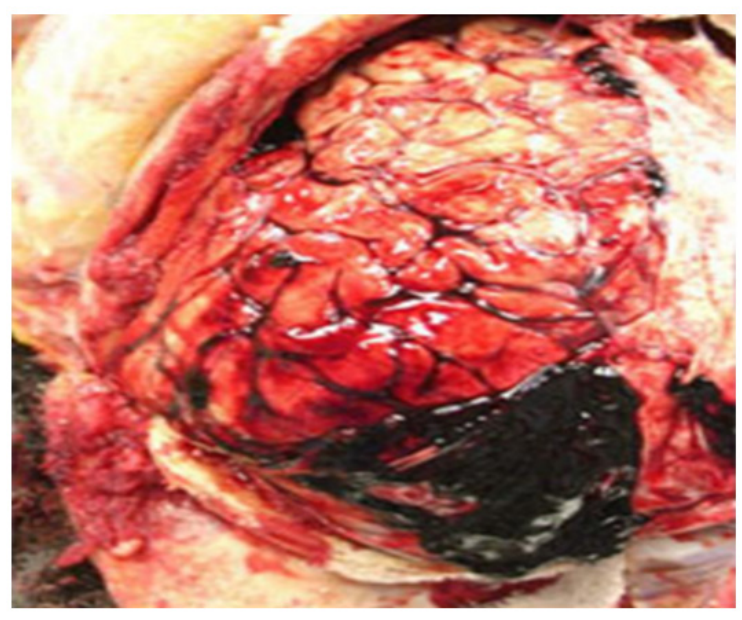

Figure I Subdural hematoma with a thickness of 2-3 $\mathrm{mm}$ in the entire posterior fossa 


\section{Discussion}

A spontaneous subdural hematoma was diagnosed as a subdural hematoma because of the presence of a severe headache in the patient, a cardiac prosthesis in his autopsy, an anticoagulant medication, and no traumatic lesion under the scalp and bone. Cases of spontaneous subdural hematomas with no predisposing factors such as trauma and alcohol are presented in the literature with the complaint like headache, nausea and vomiting. ${ }^{6}$ Spontaneous or nontraumatic acute subdural hematomas may arise from intracranial aneurysm rupture, arteriovenous malformation, hypertensive intracerebral hematoma opening to the subdural space. It has also been reported to occur in malignant metastatic patients with advanced duodenal metastases and advanced malign tumor patients. Less common are infections, coagulopathies, blood dyscrasias, myeloproliferative disorders, immun trombocytopenic purpura, hypertension, hydrocephalus decompression, anticoagulant therapy such as aspirin, heparin, warfarin, and drug use. ${ }^{7-10}$ Borger et al. ${ }^{11}$ retrospectively studied 322 patients over the age of 65 years and found that half of the patients had a history of head trauma, and three of them had anticoagulants use. ${ }^{11,12}$

Potential factors predisposing to spontaneous acute subdural hematoma are grouped under four headings;

i. The rupture of a cortical artery arising at the branch point, especially at the right angle,

ii. A small bridging artery rupture, which connects the cortical artery to the dura and connects the subdural space,

iii. Adhesions between cortical artery and arachnoid or dura mater,

iv. Cortical artery junction that attaches to dura mature and protrudes towards arachnoid.

Possibly, in any of these conditions, it is widely accepted that the artery ruptures as the result of the sudden movement of the brain during the vigorous active movement that would not be considered a head trauma. ${ }^{7,9}$

\section{Conclusion}

In this case light, it should be considered that subdural hematomas may develop spontaneously, often after aspirin, heparin, warfarin use, which may develop spontaneously even in simple movements that are not of the head trauma, resulting from sudden unexpected death of the central nervous system origin.

\section{Acknowledgement}

None.

\section{Conflict of interset}

None.

\section{References}

1. Cologlu AS. Sudden Unexpected Deaths. In: Soysal Z, Cakalir C, editiors. Forensic Medicine. Istanbul; 1999. p. 175-182.

2. Polat O, Inanici MA, Aksoy ME. Sudden Deaths of natural origin. Forensic Medicine Course Book. Nobel Medical Bookstores; 1997. p.53-59.

3. Chhiber SS, Singh JP. Acute spontaneous subdural hematoma of arterial origin: a report of four cases and review of literature. Neurol India. 2010;58(4):654-8.

4. Depreitere B, Van Calenbergh FA. Clinical comparison of non-traumatic acute subdural haematomas either related to coagulopathy or of arterial origin without coagulopathy. Neurosurgery. 2005;56:1286-92.

5. Missori P, Fenga L. Spontaneous acute subdural hematomas. ActaNeurochir(wien). 2000;142:697-701.

6. Coombs JB, Coombs BL, Chin EJ. Acute spontaneous subdural hematoma in a middle-aged adult: case report and review of the literature. $J$ Emerg Med. 2014;47(3):63-8.

7. Arai H. Acute hipertansive subdural hematoma from arteryel ruptures hortly after the onset of serebral subkortikal hemorrage: leakage of contrast medium during angiography. Stroke. 1983;14:281-285.

8. Komatsu Y, Uemura K, Yasuda S, et al. Acute subdural hemorrage of arterial origin: report of three cases. No shinkei geka. 1997;25(9):841-845.

9. McDermott M, Fleming JF, Vanderlinden RG, et al. Spontaneous arterieal subdural hematoma. Neurosurgery. 1984:14(1):13-18.

10. Ceauşu M, Dragoteanu C, Hostiuc S, et al. Sudden death due to nontraumatic subdural hematoma in a patient with a myeloproliferative disorder. Rom J Leg Med. 2015;23:251-256.

11. Borger V, Vatter H, Oszvald Á, et al. Chronic subdural haematoma in elderly patients: a retrospective analysis of 322 patients between the ages of 65-94 years. Acta Neurochir (Wien). 2012;154(9):1549-54.

12. Uysal M, Güney H, Bekar D, et al. A young patient with spontaneous subdural hematoma: case report. Journal of Contemporary Medicine. 2015;5:89-91. 